\section{Ultrasound biomicroscopic evaluation of the traumatized eyes}

MPÇ Özdal, M Mansour and J Deschênes

\author{
Abstract \\ Purpose To review causes for performing an \\ ultrasound biomicroscopic (UBM) \\ examination in traumatized eyes, to present \\ common UBM findings of 109 eyes with a \\ history of ocular trauma and to compare these \\ findings according to the type of the trauma. \\ Materials and methods A total of 109 eyes \\ with a history of mechanical ocular trauma, \\ which underwent an UBM examination \\ between December 1996 and April 2002, were \\ evaluated retrospectively. All cases were \\ classified according to the criteria of the \\ Ocular Trauma Classification Group and UBM \\ findings were reviewed. For statistical ana- \\ lyses, $\chi^{2}$ test and Fisher's exact test were used. \\ Results UBM examinations were performed \\ for the evaluation of the zonules before \\ cataract surgery $(49.5 \%)$, examination of the \\ anterior segment in the presence of media \\ opacities $(32.1 \%)$, detection of suspected ocular \\ foreign bodies $(\mathbf{1 0 . 1 \%})$ and the evaluation of \\ ocular hypotony (8.3\%). In all, 67 eyes $(61.5 \%)$ \\ had a closed-globe injury, whereas 42 (38.5\%) \\ had an open-globe injury. The most common \\ UBM findings in a closed-globe injury were \\ zonular deficiency $(64.2 \%)$, angle recession \\ (43.3\%), iridodialysis $(\mathbf{1 7 . 9 \%})$, and dislocated \\ lens (16.4\%). The most common UBM findings \\ in an open-globe injury were zonular \\ deficiency $(54.8 \%)$, iridodialysis $(26.2 \%)$, \\ peripheral anterior synechiae (PAS) (26.2\%), \\ and iridocorneal adhesion (19\%). Among the \\ common UBM findings, the angle recession \\ was significantly higher $(P<0.001)$ in closed- \\ globe injury group and PAS formation was \\ significantly higher $(P<0.05)$ in open-globe \\ injury group. \\ Conclusions UBM is particularly superior \\ to other methods in the evaluation \\ of the zonular status, angle recession, \\ cyclodialysis, and the detection of \\ small superficial and intraocular foreign \\ bodies.
}

Eye (2003) 17, 467-472. doi:10.1038/

sj.eye. 6700382

Keywords: ocular trauma; imaging; ultrasound biomicroscopy

\section{Introduction}

Ocular trauma may cause various anterior segment pathologies resulting in visual impairment or loss, such as hyphema, iridodialysis, rupture of the anterior lens capsule, lens displacement, cyclodialysis, angle recession, and intraocular foreign body. A meticulous evaluation and appropriate treatment of the traumatized eye are crucial in preventing visual impairment or loss because of ocular trauma. However, evaluation of these pathologies by clinical eye examination alone is often limited by trauma-related media opacities, hypotony, and distorted anatomy. Ultrasound biomicroscopy (UBM) allows a detailed imaging of the anterior segment up to $5 \mathrm{~mm}$ depth by using high-frequency $(50-100 \mathrm{MHz})$ transducers. ${ }^{1,2}$ This noninvasive technique enables us to visualize cornea, sclera, angle structures, iris, lens, ciliary body, peripheral choroid, and anterior vitreous even in the presence of opaque media. ${ }^{1-3}$ UBM can also detect small foreign bodies of various compositions, including those missed by computed tomography (CT ) or B-Scan ultrasound (US). ${ }^{4}$ Therefore, UBM is a helpful tool in the evaluation of the traumatized eyes by detecting anatomic disturbances and foreign body(ies) of the anterior segment.

Apart from reviewing causes for performing an UBM examination in traumatized eyes, the aim of this study was to present common UBM findings of 109 eyes with a history of mechanical ocular trauma and to compare these findings according to the type of the trauma.
Department of

Ophthalmology McGill University Health Center

Montréal, Québec, Canada

Correspondence: Dr MPÇ Özdal 2050 Blv de Maisonneuve O. \# 902,

Montréal, Québec Canada $\mathrm{H} 3 \mathrm{H} 1 \mathrm{~K} 7$

Tel: + 15149390122

Fax: + 15148431618

E-mail: pozdal@

hotmail.com

Received: 14 June 2002 Accepted: 2 August 2002 


\section{Material and methods}

In total, 109 eyes of 108 patients with a history of mechanical ocular trauma; which underwent ultrasound biomicroscopic examination between December 1996 and April 2002, were evaluated retrospectively. All cases were classified according to the criteria of the Ocular Trauma Classification Group ${ }^{5}$ and the ultrasound biomicroscopic findings were reviewed.

All patients underwent a complete ophthalmological evaluation before the UBM examination. Eyes with media opacities; which prevent the clinical examination, were also examined with a B-Scan US.

All UBM examinations were performed by the same physician (MM). Under topical anaesthesia, an eye cup filled with $2.5 \%$ methylcellulose as a coupling medium was inserted between the eyelids. An ultrasound biomicroscope with a $50 \mathrm{MHz}$ scan transducer providing a tissue resolution of $50 \mu \mathrm{m}$ and a tissue penetration depth of $5 \mathrm{~mm}$ was used to perform the UBM examinations (Humphrey Instruments, San Leandro, CA, USA). All anterior segment components including the cornea, sclera, episclera, anterior chamber, anterior chamber angle, iris, pupil, peripheral choroid, ciliary body, pars plana, lens, peripheral retina, and the anterior vitreous were examined. Complete examination was achieved with fine manual movements of the probe and also by asking the patient to look in different positions.

For statistical analyses, $\chi^{2}$ test and Fisher's exact test were used and $P$ values at 0.05 level were considered significant.

\section{Results}

Of the 108 patients, 21 were females (19.5\%) and 87 were males $(80.5 \%)$. The ages of the patients ranged from 5 to 89 years with a mean age of $49.2 \pm 19.1$ years. The main reason for UBM examination was an evaluation of the zonules before cataract surgery in 54 eyes (49.5\%). Other reasons were: more detailed examination of the anterior segment in the presence of hyphema, corneal scar, irido-corneal adhesion or cortical material in the anterior chamber (35 eyes, 32.1\%), the detection of suspected ocular foreign bodies (11 eyes, $10.1 \%$ ) and the evaluation of ocular hypotony (nine eyes, $8.3 \%$ ). The time course of imaging after trauma was variable and ranged from one day to 55 years. All examinations in the late period (6 months to 55 years) were for evaluating the zonular status.

A total of 67 of the 109 eyes (61.5\%) had a closed-globe injury, whereas $42(38.5 \%)$ had an open-globe injury. Of the eyes with a closed-globe injury, $63(57.8 \%)$ were classified as contusion, two $(1.85 \%)$ as lamellar laceration and two $(1.85 \%)$ as superficial foreign body. Of the eyes with an open-globe injury, 28 (25.7\%) had a penetrating trauma, nine $(8.3 \%)$ had an intraocular foreign body, three $(2.75 \%)$ had a perforating trauma and two $(1.85 \%)$ had a globe rupture.

The most common UBM findings of the eyes with a closed-globe injury (Table 1) were: zonular deficiency from 1-h dial to total loss (43 eyes, 64.2\%) (Figure 1), angle recession up to $360^{\circ}$ (29 eyes, $43.3 \%$ ) (Figure 2 ), iridodialysis (12 eyes, 17.9\%) (Figure 3), lens dislocation (11 eyes, 16.4\%), hyphema (nine eyes, 13.4\%), peripheral choroidal detachment (six eyes, 8.9\%) (Figure 4), peripheral anterior synechiae (PAS) up to $360^{\circ}$ (six eyes, $8.9 \%$ ) (Figure 3), ruptured anterior lens capsule (four eyes, 6\%), cyclodialysis (three eyes, 4.5\%) (Figure 4),

Table 1 The most common UBM findings of 67 eyes with a closed-globe injury

\begin{tabular}{lcc}
\hline UBM findings & Number of eyes & $\%$ \\
\hline Zonular deficiency & 43 & 64.2 \\
Angle recession & 29 & 43.3 \\
Iridodialysis & 12 & 17.9 \\
Lens dislocation & 11 & 16.4 \\
Hyphema & 9 & 13.4 \\
Peripheral choroidal detachment & 6 & 8.9 \\
Peripheral anterior synechiae & 6 & 8.9 \\
Ruptured anterior lens capsule & 4 & 6 \\
Cyclodialysis & 3 & 4.5 \\
Dislocated intraocular lens & 3 & 4.5 \\
Haemorrhagic cyst within the iris & 2 & 3 \\
Superficial foreign body & 2 & 3 \\
\hline
\end{tabular}

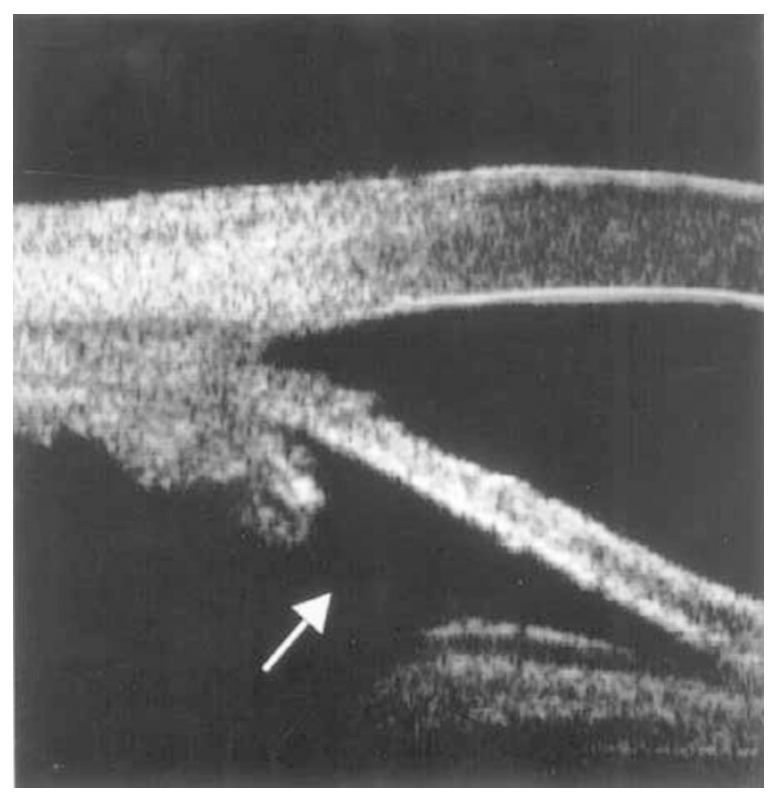

Figure 1 An ultrasound biomicroscopic image showing an area of missing zonules (arrow). 


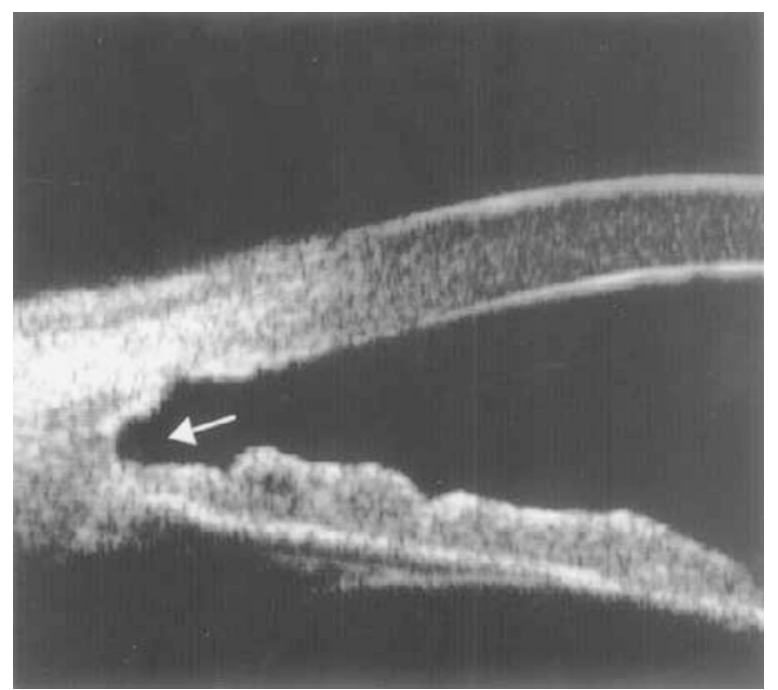

Figure 2 An ultrasound biomicroscopic image showing an angle recession (arrow).

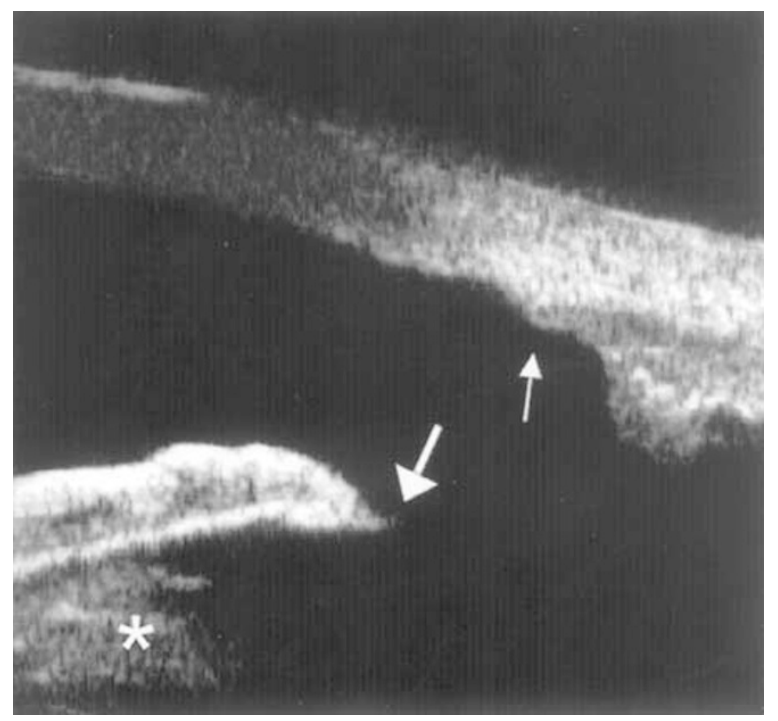

Figure 3 An ultrasound biomicroscopic image showing an iridodialysis (big arrow), peripheral anterior synechiae (small arrow), and cortical material behind the iris (asterisk).

dislocated intraocular lens (three eyes, 4.5\%) (Figure 5), haemorrhagic cyst within the iris (two eyes, 3\%) (Figure 6) and superficial foreign body (one subconjunctival, one superficial scleral: a total of two eyes, $3 \%$ ). The composition of the foreign body was metal in one and wood in the other.

The most common UBM findings of the eyes with an open-globe injury (Table 2) were zonular deficiency up to total loss of the zonules ( 23 eyes, $54.8 \%$ ), iridodialysis (11 eyes, 26.2\%), PAS (11 eyes, $26.2 \%$ ), iridocorneal adhesion (eight eyes, 19\%), intraocular foreign body (three in the ciliary body, one in the iris root, one in the iris, one in the

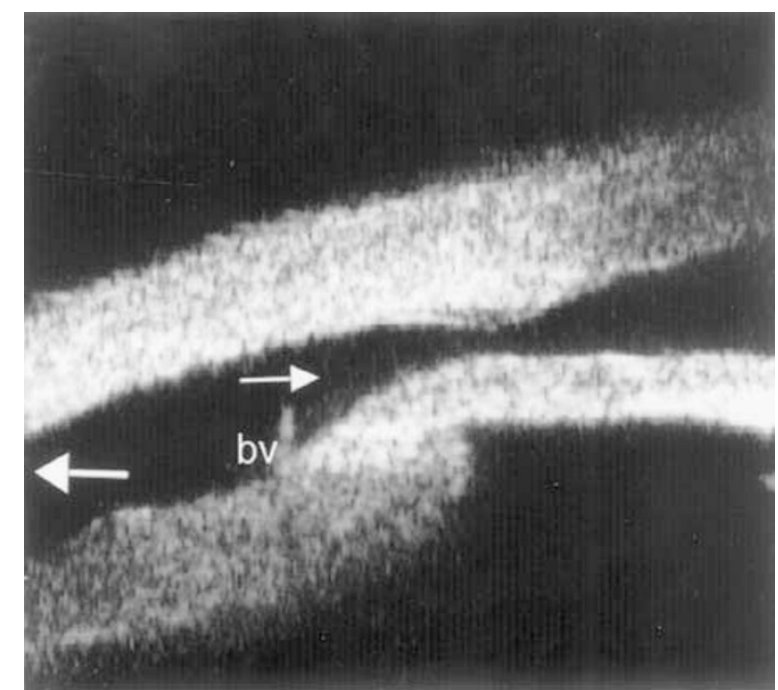

Figure 4 An ultrasound biomicroscopic image showing a cyclodialysis (small arrow) with peripheral choroidal detachment (big arrow) and small blood vessel (bv).

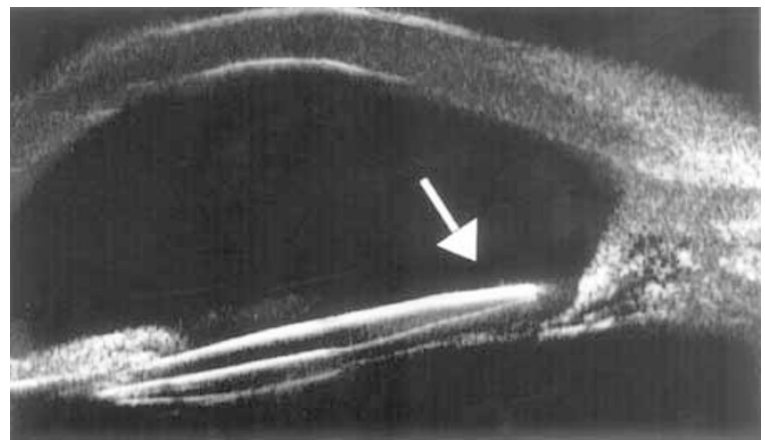

Figure 5 An ultrasound biomicroscopic image showing dislocation of posterior chamber intraocular lens in anterior chamber (arrow).

sulcus: a total of six eyes, $14.3 \%$ ) (Figure 7), angle recession up to $360^{\circ}$ (six eyes, $14.3 \%$ ), ruptured anterior lens capsule (six eyes, 14.3\%) (Figure 8), peripheral choroidal detachment (five eyes, $11.9 \%$ ), lens dislocation (five eyes, 11.9\%) (Figure 8), iris loss (five eyes, 11.9\%), cortical lens material in anterior and/or posterior chamber (five eyes, 11.9\%), hyphema (four eyes, 9.5\%), retrocorneal membrane formation (three eyes, $7.1 \%$ ), and cyclodialysis (two eyes, $4.8 \%$ ). The composition of the foreign body was metal in four, wood in one and glass in one eye.

Among the common UBM findings, the angle recession was significantly higher $(P<0.001)$ in the closed-globe injury group and PAS formation $(P<0.05)$ was significantly higher in the open-globe injury group. There was no statistically significant difference between the two groups in zonular deficiency, anterior capsule rupture, iridodialysis $(P>0.1$ for each), cyclodialysis, 


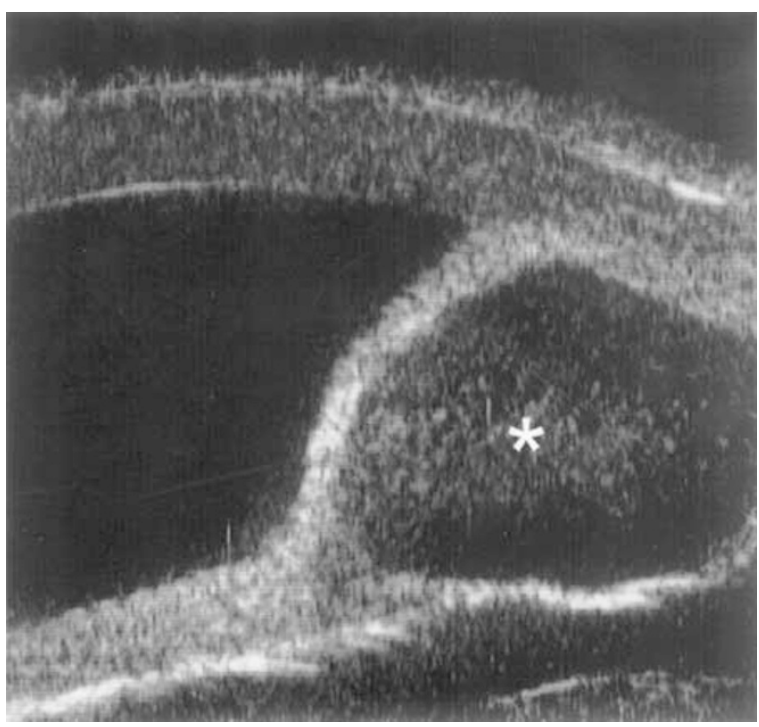

Figure 6 An ultrasound biomicroscopic image showing a haemorrhagic cyst within the iris (asterisk).

Table 2 The most common UBM findings of 42 eyes with an open-globe injury

\begin{tabular}{lcc}
\hline UBM findings & Number of eyes & $\%$ \\
\hline Zonular deficiency & 23 & 54.8 \\
Iridodialysis & 11 & 26.2 \\
Peripheral anterior synechiae & 11 & 26.2 \\
Irido-corneal adhesion & 8 & 19 \\
Intraocular foreign body & 6 & 14.3 \\
Angle recession & 6 & 14.3 \\
Ruptured anterior & 6 & 14.3 \\
lens capsule & & \\
Lens dislocation & 5 & 11.9 \\
Peripheral choroidal & 5 & 11.9 \\
detachment & & \\
Iris loss & 5 & 11.9 \\
Cortical material in & 5 & 11.9 \\
anterior/posterior chambers & & \\
Hyphema & 4 & 9.5 \\
Retrocorneal membrane formation & 3 & 7.1 \\
Cyclodialysis & 2 & 4.8 \\
\hline
\end{tabular}

hyphema, lens dislocation and peripheral choroidal detachment $(P>0.5$ for each). Iridocorneal adhesion $(P<0.001)$ was present only in the open-globe injury group.

\section{Discussion}

As a method that allows subsurface imaging of ocular structures at microscopic resolution, ${ }^{2}$ UBM can be used with different purposes in eyes exposed to a mechanical trauma. In our series, the main purpose was the evaluation of the zonular integrity before cataract

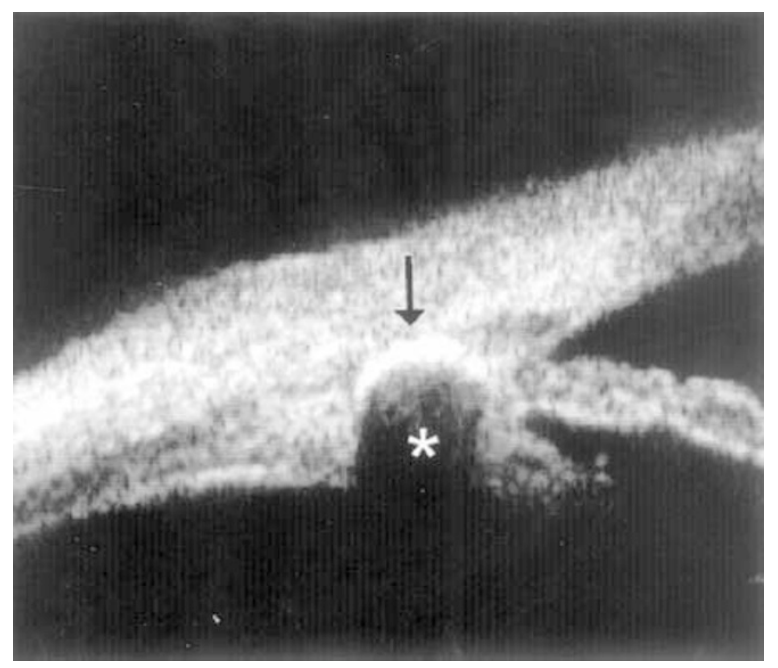

Figure 7 An ultrasound biomicroscopic image showing metallic foreign body (arrow) and its shadowing effect (asterisk) in the ciliary body.

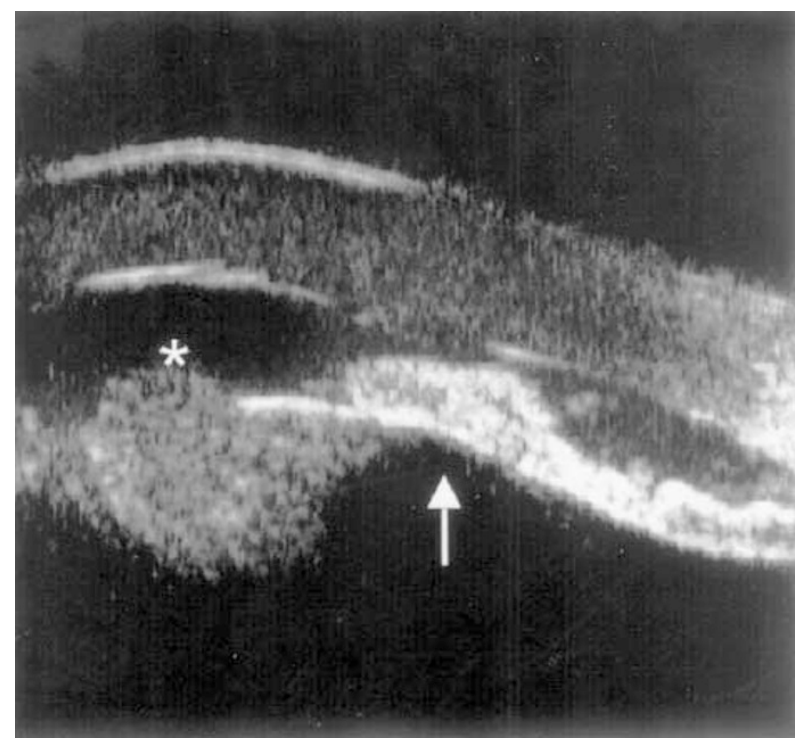

Figure 8 An ultrasound biomicroscopic image showing anteriorly dislocated lens pushing the iris (arrow) and ruptured anterior lens capsule (asterisk).

surgery $(49.5 \%)$. The evaluation of the anterior segment components, particularly in the presence of hazy ocular media $(32.1 \%)$, detection of foreign bodies $(10.1 \%)$ and evaluation of the hypotony $(8.3 \%)$, were the other purposes for performing an ultrasound biomicroscopic examination in traumatized eyes.

Zonular status is an important issue in deciding for the surgical technique that will be used. Although observing signs of increased lenticular mobility, such as phacodonesis and iridodonesis in slit-lamp examination can imply a zonular defect, the nature and extent of this defect cannot be correctly visualized. UBM enables an 
accurate determination of the presence and the amount of zonular defects, particularly when appropriate techniques are used. The zone with defect can be clearly defined in clock hours. ${ }^{6}$ Zonular deficiency was the most frequent finding in both closed-globe $(64.2 \%)$ and openglobe $(54.8 \%$ ) injuries (Figure 1). Also in a study performed by Pavlin et al, ${ }^{6}$ trauma was the most commonly identified cause of zonular defects.

As mentioned before, UBM is an excellent method to visualize angle structures. ${ }^{1-3}$ An angle recession is an anatomic pathology resulting from a traumatic tear in the anterior ciliary body, often between the circular and longitudinal muscle layers. It is often caused by a blunt trauma to the anterior segment and is associated with a retrodisplacement of the iris root. ${ }^{3,7,8}$ The frequency of this finding in our study was significantly higher in the closed-globe injury group $(P<0.001)$. On the other hand, if the ciliary body is avulsed from its normal attachment at the scleral spur, this anatomic pathology is called cyclodialysis. ${ }^{3}$ The presence of this complication was about the same in both groups of trauma (4.5-4.8\%). Cyclodialysis clefts create a direct communication between the anterior chamber and the suprachoroidal space. ${ }^{3,9,10}$ Hypotony as a result of cyclodialysis is one of the significant post-traumatic complications. ${ }^{9}$ It is caused by increased uveoscleral outflow via the suprachoroidal space and a reduction in aqueous production. ${ }^{10}$ As hypotony is followed by other complications like decreased visual acuity, reduced anterior chamber depth, choroidal detachment, and optic disc oedema, early detection and treatment of the cyclodialysis is recommended for complete recovery of the visual acuity..$^{9}$ Although the gonioscopy is very useful in the diagnosis of the angle recession and cyclodialysis clefts, it cannot be applied in recent postoperative and traumatized eyes because of the hazy ocular media, anatomic distortion, and excessive hypotony. UBM can be used to accurately differentiate these angle pathologies (Figures 2 and 4). In addition, the visualization of the supraciliary fluid and the connection from the anterior chamber to the supraciliary space confirms the diagnosis of cyclodialysis. ${ }^{3}$ UBM allows an imaging of a cyclodialysis cleft along its entire longitudinal and circumferential extent and permits an accurate determination of its location and size, regardless of gonioscopic visibility. ${ }^{7,10,11}$ UBM is a helpful tool in not only early diagnosis of the cyclodialysis, but also in determining adequate operative procedures. ${ }^{9-11}$ In a patient with traumatic hypotony, who could not be treated with an initial surgical attempt, Chan et $a l^{11}$ successfully used UBM as a guide in detecting and closing the true extent of residual cyclodialysis.

Another angle pathology observed in traumatized eyes was PAS formation (Figure 3). We realized that PAS formation was significantly more frequent (26.2 vs $8.9 \%$ ) in the open-globe injury group $(P<0.05)$.

Iridodialysis, which is a disinsertion of the iris root from its insertion into the ciliary body, can also be identified with $\mathrm{UBM}^{3,7}$ (Figure 3). The occurrence of this pathology was more often in open-globe injuries (26.2 vs $17.9 \%)$, but the difference was not statistically significant $(P>0.1)$.

Retained foreign bodies may lead to ocular morbidity, including inflammation and infection, and their detection is very important for the prognosis of the traumatized eye. Identification and localization of intraocular foreign bodies in eyes with opacified media or distorted anatomy require a radiographic or ultrasonographic evaluation.,12 X-ray, CT, magnetic resonance imaging (MRI), B-Scan US, and UBM are all important diagnostic tools in the evaluation of foreign bodies., ${ }^{4,13}$ UBM has been reported to be helpful not only in detecting the foreign body, but also in distinguishing its composition. ${ }^{12}$ It is particularly valuable in the detection of small and nonmetallic foreign bodies. Foreign bodies made of wood or plastic may appear hypodense on CT and can be confused with air. Additionally, determining whether foreign bodies surrounding the scleral wall are intraocular or extraocular can be difficult with CT. Furthermore, the minimum detectable size of an ocular foreign body by CT depends on the object's composition. On the other hand, MRI, besides being an expensive examination, is contraindicated in cases with suspected metallic foreign bodies. ${ }^{4}$ The exact location of the foreign body, particularly when very small, requires high resolution. Although the $10 \mathrm{MHz}$ B-Scan US is a very useful test after ocular trauma, high-frequency $(50 \mathrm{MHz})$ ultrasound is reported to be much better in showing the exact location of the foreign body and the condition of surrounding tissues $^{12,14}$ (Figure 7). Deramo et al ${ }^{4}$ reported that UBM could detect small foreign bodies of various compositions, including those missed by CT or US. They recommended the UBM as a useful adjunct to CT and US in the detection and localization of small superficial and intraocular foreign bodies. ${ }^{4}$ Laroche et al ${ }^{12}$ suggested UBM as the preferred initial imaging step in searching for an occult foreign body. Barash et l $^{13}$ emphasized that CT was insufficient to detect very small foreign bodies and their relationship to the surrounding tissue, compared with UBM. In our study, two with superficial and six with intraocular, a total of eight eyes (7.3\%) with foreign body were detected by UBM.

Lens dislocation (Figure 8), hyphema, peripheral choroidal detachment (Figure 4), and ruptured anterior lens capsule (Figure 8) were the other common findings of the UBM examination in mechanically injured eyes. The presence of these findings revealed no statistically significant difference between the two groups of trauma 
$(P>0.1$ to $P>0.5)$. Iridocorneal adhesion $(P<0.001)$ was present only in the open-globe injury group.

As a safe and noninvasive technique, UBM can be used in cases with closed-globe injuries, healed perforated corneas and also in cases with open-globe injuries when necessary. For the evaluation of open-globe injuries, the eye cup should be inserted so that minimal pressure is placed on the globe and should be filled with sterile coupling medium. ${ }^{10}$ Other than being safe and noninvasive, greater accuracy and ease of performance are its advantages over conventional methods. ${ }^{13}$

However, being limited with only the anterior segment evaluation is the main disadvantage of UBM in evaluating traumatized eyes. UBM is especially superior to other methods in the evaluation of the zonular status, angle recession, cyclodialysis and the detection of small superficial, and intraocular foreign bodies.

\section{Acknowledgement}

This paper does not serve the financial or proprietary interests of any individuals nor institutions.

\section{References}

1 Pavlin CJ. Interpreting technology. Practical application of ultrasound biomicroscopy. Can J Ophthalmol 1995; 30(4): 225-229.

2 Pavlin CJ, Harasiewicz K, Sherar MD, Foster FS. Clinical use of ultrasound biomicroscopy. Ophthalmology 1991; 98: 287-295.

3 Liebmann JM, Ritch R, Esaki K. Ophthalmic imaging and diagnostics. Ultrasound biomicroscopy. Ophthalmol Clin North Am 1998; 11(3): 421-433.
4 Deramo VA, Gaurav KS, Baumal CR, Fineman MS, Correa $\mathrm{ZM}$, Benson WE et al. Ultrasound biomicroscopy as a tool for detecting and localizing occult foreign bodies after ocular trauma. Ophthalmology 1999; 106: 301-305.

5 Pieramici DJ, Sternberg P, Aaberg TM, Bridges WZ, Capone A, Cardillo JA et al. The Ocular Trauma Classification Group. A system for classifying mechanical injuries of the eye (globe). Am J Ophthalmol 1997; 123: 820-831.

6 Pavlin CJ, Buys Y, Pathmanathan T. Imaging zonular abnormalities using ultrasound biomicroscopy. Arch Ophthalmol 1998; 116: 854-857.

7 Berinstein DM, Gentile RC, Sidoti PA, Stegman Z, Tello C, Liebmann JM et al. Ultrasound biomicroscopy in anterior ocular trauma. Ophthalmic Surg Lasers 1997; 28: 201-207.

8 Wolff SM, Zimmerman LE. Chronic secondary glaucoma: associated with retrodisplacement of iris root and deepening of the anterior chamber angle secondary to contusion. Am J Ophthalmol 1962; 54: 547-550.

9 Park M, Kondo T. Ultrasound biomicroscopic findings in a case of cyclodialysis. Ophthalmologica 1998; 212: 194-197.

10 Gentile RC, Pavlin CJ, Liebmann JM, Easterbrook M, Tello C, Foster FS et al. Diagnosis of traumatic cyclodialysis by ultrasound biomicroscopy. Ophthalmic Surg Lasers 1996; 27: 97-105.

11 Chan TKJ, Talbot JF, Rennie IG, Longstaff S, Desai SP. The application of ultrasonic biomicroscopy in the management of traumatic hypotony. Eye 2000; 14(Part 5): 805-807.

12 Laroche D, Ishikawa H, Greenfield D, Liebmann JM, Ritch R. Ultrasound biomicroscopic localization and evaluation of intraocular foreign bodies. Acta Ophthalmol Scand 1998; 76: 491-495.

13 Barash D, Goldenberg-Cohen N, Tzadok D, Lifshitz T, Yassour Y, Weinberger D. Ultrasound biomicroscopic detection of anterior ocular segment foreign body after trauma. Am J Ophthalmol 1998; 126: 197-202.

14 Nouby-Mahmoud G, Silverman RH, Coleman DJ. Using high-frequency ultrasound to characterize intraocular foreign bodies. Ophthalmic Surg 1993; 24: 94-99. 